AperTO - Archivio Istituzionale Open Access dell'Università di Torino

\title{
Rediscovery of Good-Turing estimators via Bayesian nonparametrics
}

\section{This is the author's manuscript}

Original Citation:

Availability:

This version is available http://hdl.handle.net/2318/1591184

since 2016-09-03T11:55:58Z

Published version:

DOI:10.1111/biom.12366

Terms of use:

Open Access

Anyone can freely access the full text of works made available as "Open Access". Works made available under a Creative Commons license can be used according to the terms and conditions of said license. Use of all other works requires consent of the right holder (author or publisher) if not exempted from copyright protection by the applicable law. 
Summary. The problem of estimating discovery probabilities originated in the context of statistical ecolo years it has become popular due to its frequent appearance in challenging applications arising in genetic linguistics, designs of experiments, machine learning, etc. A full range of statistical approaches, parametric ar as well as frequentist and Bayesian, has been proposed for estimating discovery probabilities. In this artic the relationships between the celebrated Good-Turing approach, which is a frequentist nonparametric appro the 1940s, and a Bayesian nonparametric approach recently introduced in the literature. Specifically, unde of a two parameter Poisson-Dirichlet prior, we show that Bayesian nonparametric estimators of discovery asymptotically equivalent, for a large sample size, to suitably smoothed Good-Turing estimators. As a by result, we introduce and investigate a methodology for deriving exact and asymptotic credible intervals with the Bayesian nonparametric estimators of discovery probabilities. The proposed methodology is illus comprehensive simulation study and the analysis of Expressed Sequence Tags data generated by sequenc complementary DNA library.

KEY WORDS: Asymptotic equivalence; Bayesian nonparametrics; Credible intervals; Discovery probabilit quence Tags; Good-Toulmin estimator; Good-Turing estimator; Smoothing technique; Two parameter Poisso

\section{Introduction}

Consider a population of individuals $\left(X_{i}\right)_{i \geq 1}$ belonging to an (ideally) infinite number of species $\left(X_{i}^{*}\right)_{i \geq 1}$ with unknown proportions $\left(p_{i}\right)_{i \geq 1}$. Given an initial observed sample of size $n$, a quantity of practical interest is the probability $D_{n, m}(l)$ of observing at the $(n+m+1)$-th drawn a species with frequency $l \geq 0$ in the enlarged sample of size $n+m$, with the additional sample being unobserved. Formally, if $N_{i, n+m}$ denotes the frequency of $X_{i}^{*}$ in the enlarged sample, then

$$
D_{n, m}(l)=\sum_{i \geq 1} p_{i} \mathbb{1}_{\{l\}}\left(N_{i, n+m}\right) .
$$

Clearly $D_{n, m}(0)$ corresponds to the proportion of yet unobserved species or, equivalently, the probability of discovering a new species at the $(n+m+1)$-th drawn. The random probability $(1)$ is typically referred to as the $(m ; l)$-discovery. While the $(0 ; l)$-discovery is of interest for estimating the probability of discovering new species or rare species, the $(m ; l)$-discovery is typically of interest in decision problems regarding the size of the additional sample to collect.

A full range of statistical approaches, parametric and nonparametric as well as frequentist and Bayesian, have been proposed for estimating $D_{n, m}(l)$. These approaches have originally found applications in ecology, and their importance has grown considerably in recent years, driven by challenging applications arising in genetics, bioinformatics, linguistics, designs of experiments, machine learning, etc. See Bunge and Fitzpatrick (1993) and Bunge et al. (2014) for comprehensive re- views. In this article we investigate the relat two approaches for estimating $D_{n, m}(l)$ : (i) the parametric approach which appeared in the Good (1953), and first developed by Alan M ing J. Good during their collaboration at $\mathrm{H}$ the 1940s; (ii) the Bayesian nonparametric a introduced by Lijoi et al. (2007) and Favaro order to state our main contributions, we $b$ relevant aspects of these two nonparametric

\subsection{The Good-Turing Approach}

Let $\mathscr{H}$ be a parametric statistical hypoth that is $\mathscr{H}$ determines the species composi ulation by specifying a distribution funct and with a finite number of unknown paral $\left(X_{1}, \ldots, X_{n}\right)$ be a random sample from $\mathscr{H}$, by $M_{l, n}$ the number of species with frequency ing to Good (1953), an estimator of $D_{n, 0}(l$ $(l+1) \mathbb{E}_{\mathscr{H}}\left[M_{l+1, n+1}\right] /(n+1)$, where $\mathbb{E}_{\mathscr{H}}$ deno value with respect to the distribution func $\mathscr{H}$. For any $m \geq 1$ let us consider the served sample $\left(X_{n+1}, \ldots, X_{n+m}\right)$, and define ? ing to Good and Toulmin (1956), an estim. $\check{\mathcal{D}}_{n, m}(0 ; \mathscr{H})=\sum_{i \geq 1}(-\gamma)^{i-1} i \mathbb{E}_{\mathscr{H}}\left[M_{i, n+m}\right] / n$. No ple, $\mathbb{E}_{\mathscr{H}}\left[M_{l+1, n+1}\right]$ and $\mathbb{E}_{\mathscr{H}}\left[M_{i, n+m}\right]$ do not dep observed sample, unless the parameters char estimated using such a sample. Several ex thoroughly discussed in Good (1953) and, mention the Zipf-type distributions and the son distributions. 
The large $n$ approximate estimator (2) is known as the Good-Turing estimator. A similar large $n$ approximation was proposed in Good and Toulmin (1956) for $\check{\mathcal{D}}_{n, m}(0 ; \mathscr{H})$. Specifically,

$$
\check{\mathcal{D}}_{n, m}(0 ; \mathscr{H}) \bumpeq \check{\mathcal{D}}_{n, m}(0)=\frac{1}{n} \sum_{i \geq 1}(-\gamma)^{i-1} i m_{i, n} .
$$

$\check{\mathcal{D}}_{n, m}(0)$ is known as the Good-Toulmin estimator for the ( $m ; 0)$-discovery. As observed by Good and Toulmin (1956), due to the alternating sign of the series which appears in the estimator (3), if $\gamma$ is large then $\check{\mathcal{D}}_{n, m}(0)$ can yield inadmissible estimates. This instability arises even for values of $m$ moderately larger than $n$, typically $m$ greater than $n$ is enough for it to appear.

A peculiar feature of $\check{\mathcal{D}}_{n, 0}(l)$ is that it depends on $m_{l+1, n}$, and not on $m_{l, n}$ as one would intuitively expect for an estimator of the $(0 ; l)$-discovery. Such a feature, combined with the irregular behavior of the $m_{l, n}$ 's for large $l$, makes $\check{\mathcal{D}}_{n, 0}(l)$ a sensible approximation only if $l$ is sufficiently small with respect to $n$. Indeed for some large $l$ one might observe that $m_{l, n}>0$ and $m_{l+1, n}=0$, which provides the absurd estimate $\check{\mathcal{D}}_{n, 0}(l)=0$, or that $m_{l, n}<m_{l+1, n}$ although the overall observed trend for $m_{l, n}$ is to decrease as $l$ increases. In order to overcome these drawbacks Good (1953) suggested to smooth the irregular series of $m_{l, n}$ 's into a more regular series to be used as a proxy. If $m_{l, n}^{\prime}$ 's are the smoothed $m_{l, n}$ 's with respect to a smoothing rule $\mathscr{S}$, then $\check{\mathcal{D}}_{n, 0}(l ; \mathscr{S})=(l+1) m_{l+1, n}^{\prime} / n$ is a more accurate approximation than $\check{\mathcal{D}}_{n, 0}(l)$. Common smoothing rules consider $m_{l, n}^{\prime}$, as a function of $l$, to be approximately parabolic or, alternatively, $m_{l, n}^{\prime}$ to be a certain proportion of the number of species in $\boldsymbol{X}_{n}$. An alternative method assumes $\mathscr{H}$ to be selected from a superpopulation with an assigned distribution. This flexible method was hinted at in Good (1953) and then left as an open problem.

\subsection{The Bayesian Nonparametric Approach}

The approach in Lijoi et al. (2007) and Favaro et al. (2012) is based on the randomization of $p_{i}$ 's. This is somehow reminiscent of the superpopulation smoothing hinted at by Good (1953). Specifically, let $P=\sum_{i \geq 1} p_{i} \delta_{X_{i}^{*}}$ be a discrete random probability measure, namely $\left(p_{i}\right)_{i \geq 1}$ are nonnegative random weights such that $\sum_{i \geq 1} p_{i}=1$ almost surely, and $\left(X_{i}^{*}\right)_{i \geq 1}$ are random locations independent of $\left(p_{i}\right)_{i \geq 1}$ and independent and identically distributed as a nonatomic distribution. The sample $\boldsymbol{X}_{n}$ is drawn from a population with species composition determined by $P$, i.e.,

$$
X_{i} \mid P \stackrel{\text { iid }}{\sim} P \quad i=1, \ldots, n \quad P \sim \mathscr{P}
$$

Under the framework (4), and with prior, Lijoi et al. (2007) and Fava rived a Bayesian nonparametric est discovery. Specifically, let $\boldsymbol{X}_{n}$ be a $\mathrm{s}$ turing $K_{n}=k_{n}$ species with correspon $\left(M_{1, n}, \ldots, M_{n, n}\right)=\left(m_{1, n}, \ldots, m_{n, n}\right)$. Fro Lijoi et al. (2007), the Bayesian nonpa $D_{n, m}(0)$, with respect to a squared loss

$$
\hat{\mathcal{D}}_{n, m}(0)=\frac{\theta+\sigma k_{n}}{\theta+n} \frac{(\theta+n}{(\theta+n}
$$

for any $m \geq 0$, where $(a)_{n}=\prod_{0 \leq i \leq n-1}(c$ $(a)_{0} \equiv 1$. For any $m \geq 0$, let $\left(\bar{X}_{n+1}, \ldots\right.$ tional unobserved sample from $P_{\sigma, \theta}$. Ac in Favaro et al. (2012), the Bayesian no of $D_{n, m}(l)$, with respect to a squared lo

$$
\begin{aligned}
\hat{\mathcal{D}}_{n, m}(l)= & \sum_{i=1}^{l}\left(\begin{array}{c}
m \\
l-i
\end{array}\right) m_{i, n}(i-\sigma)_{l+1-i} \\
& \left.+(1-\sigma)_{l}\left(\begin{array}{c}
m \\
l
\end{array}\right)\left(\theta+\sigma k_{n}\right)\right)
\end{aligned}
$$

for any $l=1, \ldots, n+m$. According to $\mathrm{t}$ (5) and (6), the Bayesian nonparametri table advantages with respect to the $G$ (i) it leads directly to exact estimators, of large $n$ approximations; (ii) $\hat{\mathcal{D}}_{n, 0}(l)$ i $m_{l, n}$, and not of $m_{l+1, n}$, thus avoiding the ing techniques to prevent absurd estima irregular behavior of the $m_{l, n}$ 's.

\subsection{Contributions of the Paper and}

Let $a_{n} \simeq b_{n}$ mean that $\lim _{n \rightarrow+\infty} a_{n} / b_{n}=$ are asymptotically equivalent as $n$ ten article we show that the Bayesian $\mathrm{n}$ tor $\hat{\mathcal{D}}_{n, 0}(l)$ is asymptotically equivalen $n$ tends to infinity, to a Good-Turing e: smoothed frequency counts. More preci we show that $\hat{\mathcal{D}}_{n, 0}(l) \simeq \check{\mathcal{D}}_{n, 0}\left(l ; \mathscr{S}_{\mathrm{PD}}\right)$ as is a smoothing rule such that $m_{l, n}$ is sm

$$
m_{l, n}^{\prime}=\frac{\sigma(1-\sigma)_{l-1}}{l !} k
$$

While smoothing techniques were intro as an ad hoc tool for post processing improve the performance of $\check{\mathcal{D}}_{n, 0}(l)$, our 
Good-Turing estimator $\mathcal{D}_{m, 0}(l)$ in which $m_{l+1, m}$ is replaced by a smoothed version, via $\mathscr{S}_{\mathrm{PD}}$, of the Bayesian nonparametric estimator $\hat{\mathcal{M}}_{n, m}(l+1)$ of the number of species with frequency $l$ in the enlarged sample. As a by-product of this result, we introduce a methodology for deriving large $m$ asymptotic credible intervals for $\hat{\mathcal{D}}_{n, m}(l)$, thus completing the study in Lijoi et al. (2007) and Favaro et al. (2012). While the $\operatorname{PD}(\sigma, \theta)$ prior leads to an explicit expression for the posterior distribution of $D_{n, m}(l)$, this expression involve combinatorial coefficients whose evaluation for large $m$ is cumbersome, thus preventing its implementation for determining exact credible intervals. Our methodology thus provides a fundamental tool in many situations of practical interest, arising especially in genomics, where $m$ is required to be very large and only a small portion of the population is sampled.

Our results are illustrated through a simulation study and the analysis of Expressed Sequence Tags (ESTs) data generated by sequencing a benchmark complementary DNA (cDNA) library. By means of a simulation study, we compare $\check{\mathcal{D}}_{n, 0}\left(l ; \mathscr{S}_{\mathrm{PD}}\right)$ with smoothed Good-Turing estimators obtained by using the Poisson smoothing and a smoothing technique in Sampson (2001). Simulated data are generated from the Zeta distribution, whose power law behavior is common in numerous applications. In order to detect the effects of different smoothing techniques, we compare the smoothed GoodTuring estimators with $\check{\mathcal{D}}_{n, 0}(l)$ and $\hat{\mathcal{D}}_{n, 0}(l)$. A second numerical illustration is devoted to the large $m$ asymptotic credible intervals for the Bayesian nonparametric estimator $\hat{\mathcal{D}}_{n, m}(l)$. Using ESTs data, we compare asymptotic confidence intervals for the Good-Toulmin estimator $\check{\mathcal{D}}_{n, m}(0)$ with asymptotic credible intervals for its Bayesian nonparametric counterpart $\hat{\mathcal{D}}_{n, m}(0)$. This study completes the numerical illustration presented in Favaro et al. (2009) and Favaro et al. (2012) on the same ESTs data.

In Section 2, we present and discuss the asymptotic equivalence between the Good-Turing approach and the Bayesian nonparametric approach under the assumption of the $\operatorname{PD}(\sigma, \theta)$ prior. As a by-product of this asymptotic analysis, in Section 3 we introduce a methodology for associating large $m$ asymptotic credible intervals to $\hat{\mathcal{D}}_{n, m}(l)$. Section 4 contains numerical illustrations. Proofs of our results, as well as related additional materials, and the Matlab code for computing asymptotic credible intervals are available as web-based supplementary materials.

\section{Good Turing Estimators via Bayesian Nonparametrics}

Under a $\operatorname{PD}(\sigma, \theta)$ prior, the most notable difference between the Good-Turing estimator and its Bayesian nonparametric counterpart can be traced back to the different use of the in-
$\mathcal{D}_{n, m}(l)$ and $\mathcal{D}_{m, 0}(l)$. With a slight abuse of $\mathrm{n}$ out this section we write $X \mid Y$ to denote a whose distribution coincides with the condit of $X$ given $Y$.

\subsection{Large $n$ Asymptotic Equivalences for $\mathcal{Z}$}

We start by recalling the predictive distrik izing $P_{\sigma, \theta}$. Let $\boldsymbol{X}_{n}$ be a sample of size $n$ $k_{n}$ species $X_{1}^{*}, \ldots, X_{K_{n}}^{*}$ with frequencies $\left(n_{1, n}, \ldots, n_{k_{n}, n}\right)$. According to the de Finett theorem, $\boldsymbol{X}_{n}$ is part of an exchangeable seque distribution has been characterized by Pitm lows

$$
\mathbb{P}\left[X_{n+1} \in \cdot \mid \boldsymbol{X}_{n}\right]=\frac{\theta+\sigma k_{n}}{\theta+n} v_{0}(\cdot)+\frac{1}{\theta+n} \sum_{i=1}^{k_{n}}
$$

with $v_{0}$ being a nonatomic probability mea tional probability (8) is referred to as the bution of $P_{\sigma, \theta}$. Note that $\hat{\mathcal{D}}_{n, 0}(l)$ can be re deed from (5) and (6) one has $\hat{\mathcal{D}}_{n, 0}(0)=(\theta+$ $\hat{\mathcal{D}}_{n, 0}(l)=(l-\sigma) m_{l, n} /(\theta+n)$, respectively. Se for details on (8), and on the joint distrib $\left(N_{1, n}, \ldots, N_{K_{n}, n}\right)$ induced by $(8)$.

The asymptotic equivalence betwee $\check{D}_{n, 0}\left(l ; \mathscr{S}_{\mathrm{PD}}\right)$ relies on an interesting in the large $n$ asymptotic behaviors of $K_{n}$ a $\operatorname{PD}(\sigma, \theta)$ prior. Specifically, let $A_{n} \stackrel{\text { a.s. }}{\simeq} B_{n}$ as that $\lim _{n \rightarrow+\infty} A_{n} / B_{n}=1$ almost surely, name almost surely asymptotically equivalent as $n$ By a direct application of Theorem 3.8 anc Pitman (2006), one obtains the asymptotic

$$
M_{l, n} \stackrel{\text { a.s. }}{\simeq} \frac{\sigma(1-\sigma)_{l-1}}{l !} K_{n}
$$

as $n \rightarrow+\infty$. In other terms, under a $\operatorname{PD}(c$ sample size $n$ tends to infinity the number frequency $l$ becomes a proportion $\sigma(1-\sigma)_{l}$. number of species. We refer to the web appen details on (9). The next theorem combines ( 8 to establish the asymptotic equivalence bet $\check{D}_{n, 0}\left(l ; \mathscr{S}_{\mathrm{PD}}\right)$.

TheOREM 1. Let $\boldsymbol{X}_{n}$ be a sample of featuring $K_{n}=k_{n}$ species with corresponding 
the frequency count $m_{l, n}$ by taking the proportion $\sigma(1-$ $\sigma)_{l-1} / l$ ! of $k_{n}$. Such a smoothing rule is somehow related to the Poisson smoothing $\mathscr{S}_{\text {Poi }}$, originally introduced by Good (1953), in which the frequency count $m_{l, n}$ is approximately equal to a proportion $\mathrm{e}^{-\lambda} \lambda^{\tau+l-1} /(\tau+l-1)$ ! of $k_{n}$, for any $\lambda>0$ and $\tau \geq 0$ such that $\sum_{l \geq 0} \check{D}_{n, 0}\left(l ; \mathscr{S}_{\text {Poi }}\right)=1$. See Chapter 2 in Engen (1978) for a common example of Poisson smoothing where $\tau=1$ and $\lambda=n / k_{n}$. In particular $\mathscr{S}_{\mathrm{PD}}$ is related to the Poisson smoothing corresponding to the choice $\tau=0$ and to a suitable randomization of the parameter $\lambda$. Specifically, let us denote by $P_{\lambda}$ a discrete random variable with distribution $\mathbb{P}\left[P_{\lambda}=l\right]=\mathrm{e}^{-\lambda} \lambda^{l-1} /(l-1)$ !, that is the Poisson smoothing with $\tau=0$ and $\lambda>0$. If $G_{a, b}$ is Gamma random variable with parameter $(a, b)$ and $L_{\sigma}$ is a discrete random variable with distribution $\mathbb{P}\left[L_{\sigma}=l\right]=\sigma(1-\sigma)_{l-1} / l$ !, then according to Devroye (1993) $L_{\sigma} \stackrel{\mathrm{d}}{=} 1+P_{G_{1,1} G_{1,1-\sigma} / G_{1, \sigma}}$ where $G_{1,1}, G_{1,1-\sigma}$ and $G_{1, \sigma}$ are mutually independent.

A peculiar feature of the smoothing rule $\mathscr{S}_{\mathrm{PD}}$ is that it depends only on $\sigma \in(0,1)$. This is because $\mathscr{S}_{\mathrm{PD}}$ is obtained by suitably combining (9), which does not depend of the parameter $\theta$, with other two large $n$ asymptotic equivalences independent of $\theta$, namely: (i) $\hat{\mathcal{D}}_{n, 0}(0) \simeq \sigma k_{n} / n$ and ii) $\hat{\mathcal{D}}_{n, 0}(l) \simeq(l-\sigma) m_{l, n} / n$. We conjecture that these asymptotic equivalences, as well as (9), hold for a more general class of priors considered in Lijoi et al. (2007) and Favaro et al. (2012). This is the class of Gibbs-type priors introduced by Pitman (2003) and including two of the most commonly used nonparametric priors, i.e., the $\operatorname{PD}(\sigma, \theta)$ prior and the normalized generalized Gamma prior. See De Blasi et al. (2015) for details. In other terms, our conjecture is that Theorem 1 holds for any Gibbs-type prior, that is the smoothing rule $\mathscr{S}_{\mathrm{PD}}$ is invariant with respect to the choice of any prior in the Gibbs class. Intuitively, different smoothing rules for different Gibbs-type priors, if they exist, necessarily require to investigate the high-order large $n$ asymptotic behavior of $\hat{\mathcal{D}}_{n, 0}(l)$, and then combine it with a corresponding refinement of the asymptotic equivalence in (9). Work on this is ongoing.

\subsection{Large $m$ Asymptotic Equivalences for $\hat{\mathcal{D}}_{n, m}(l)$}

Let $\boldsymbol{X}_{n}$ be a sample of size $n$ from $P_{\sigma, \theta}$ featuring $K_{n}=k_{n}$ species with frequency counts $\left(M_{1, n}, \ldots, M_{n, n}\right)=\left(m_{1, n}, \ldots, m_{n, n}\right)$. For any $m \geq 1$ let $\left(X_{n+1}, \ldots, X_{n+m}\right)$ be an additional unobserved sample. Let $K_{m}^{(n)}$ be the number of new species in $\left(X_{n+1}, \ldots, X_{n+m}\right)$ and let $M_{l, m}^{(n)}$ denote the number of species with frequency $l$ in $\left(X_{1}, \ldots, X_{n+m}\right)$. Since the additional sample is assumed to be not observed, let us introduce a randomized version of $\hat{\mathcal{D}}_{n+m, 0}(0)$ and $\hat{D}_{n+m, 0}(l)$ as

$$
D_{0, m}^{(n)}=\frac{\theta+\sigma k_{n}+\sigma K_{m}^{(n)}}{\theta+n+m}
$$

according to the expression $(6),\left(K_{n}, N\right.$ ficient statistic for $\hat{\mathcal{D}}_{n, m}(l)$ and, therefo $D_{n, m}^{(n)}(l) \mid \boldsymbol{X}_{n}$ takes on the interpretation bution, with respect to $\boldsymbol{X}_{n}$, of the $(m ; l$

By means of the identities introdu the distribution of $D_{0, m}^{(n)} \mid \boldsymbol{X}_{n}$ and $D_{n, m}^{(n)}(l$ distribution of $K_{m}^{(n)} \mid \boldsymbol{X}_{n}$ and $M_{l, m}^{(n)} \mid \boldsymbol{X}_{n}$, re been obtained in Lijoi et al. (2007) an See the web appendix for details on particular, Proposition 1 in Favaro et a

$$
\hat{\mathcal{K}}_{n, m}=\mathbb{E}\left[K_{m}^{(n)} \mid \boldsymbol{X}_{n}\right]=\frac{\left(\theta / \sigma+k_{n}\right)}{(\theta+n)_{m}}((\theta+
$$

which is the Bayesian nonparametric e to a squared loss function, of $K_{m}^{(n)}$. Fur $1, \ldots, n+m$, Proposition 7 in Favaro et

$$
\begin{aligned}
\hat{\mathcal{M}}_{n, m}(l)= & \mathbb{E}\left[M_{l, m}^{(n)} \mid \boldsymbol{X}_{n}\right] \\
= & \sum_{i=1}^{l}\left(\begin{array}{c}
m \\
l-i
\end{array}\right) m_{i, n}(i-\sigma)_{l-i} \\
& +(1-\sigma)_{l-1}\left(\begin{array}{c}
m \\
l
\end{array}\right)\left(\theta+\sigma k^{\prime}\right.
\end{aligned}
$$

which is the Bayesian nonparametric spect to a squared loss function, of means of (11) and (12) one obtains $\hat{\mathcal{D}}$ $\left(\theta+\sigma k_{n}+\sigma \hat{\mathcal{K}}_{n, m}\right) /(\theta+n+m)$ and $(l-\sigma) \hat{\mathcal{M}}_{n, m}(l) /(\theta+n+m)$, which provi resentation for the estimators of the $(m ; l)$-discovery, respectively.

Similarly to Theorem 1 , an asymptot $\hat{\mathcal{D}}_{n, m}(l)$ and $\check{\mathcal{D}}_{m, 0}(l)$ relies on the interp $m$ asymptotic behaviors of the random $M_{l, m}^{(n)} \mid \boldsymbol{X}_{n}$. Specifically, for any $n \geq 1$, b of Proposition 2 in Favaro et al. (2009 Gnedin et al. (2007) one obtains the equivalence

$$
M_{l, m}^{(n)} \mid \boldsymbol{X}_{n} \stackrel{\text { a.s. }}{\simeq} \frac{\sigma(1-\sigma)_{l-1}}{l !}
$$

as $m \rightarrow+\infty$. In other terms, under a $\mathrm{P}$ $m$ asymptotic equivalence between $M_{l, m}^{(n}$ incides with the large $n$ asymptotic equ and $K_{n}$. We refer to the web appendix 


$$
\hat{\mathcal{D}}_{n, m}(l) \simeq(l+1) \frac{\mathcal{M}_{n, m}(l+1)}{m} \simeq(l+1) \frac{\frac{\frac{(l+1) !}{\left(l+\mathcal{K}_{n, m}\right.}}{m}}{m}
$$

Besides discovery probabilities one is also interested in cumulative discovery probabilities, which are generalizations of the $(m ; l)$-discovery defined as follows. For any $\tau \geq$ 1 , let $\left\{l_{1}, \ldots, l_{\tau}\right\}$ be a collection of distinct indexes such that $l_{i} \in\{0,1, \ldots, n+m\}$ for any $i=1, \ldots, \tau$. We define the $\left(m ; l_{1}, \ldots, l_{\tau}\right)$-discovery as the cumulative discovery probability $D_{n, m}\left(l_{1}, \ldots, l_{\tau}\right)=\sum_{1 \leq i \leq \tau} D_{n, m}\left(l_{i}\right)$. Hence, the Bayesian nonparametric estimator of $\left(m ; l_{1}, \ldots, l_{\tau}\right)$-discovery is

$$
\hat{\mathcal{D}}_{n, m}\left(l_{1}, \ldots, l_{\tau}\right)=\sum_{i=1}^{\tau} \hat{\mathcal{D}}_{n, m}\left(l_{i}\right) .
$$

Such a generalization of the $(m ; l)$-discovery is mainly motivated by several applications of practical interest in which one aims at estimating the probability of discovering the so-called rare species. Specifically, these are species not yet observed or observed with a frequency smaller than a certain threshold $\tau$. Of course, large $n$ and large $m$ asymptotic equivalences for the estimator $\hat{\mathcal{D}}_{n, m}\left(l_{1}, \ldots, l_{\tau}\right)$ follow by a direct application of Theorem 1 and Theorem 2, respectively.

\section{Credible Intervals for $\hat{\mathcal{D}}_{n, m}\left(l_{1}, \ldots, l_{\tau}\right)$}

While deriving the estimator $\hat{\mathcal{D}}_{n, m}(l)$, Lijoi et al. (2007) and Favaro et al. (2012) did not consider the problem of associating a measure of uncertainty to $\hat{\mathcal{D}}_{n, m}(l)$. Such a problem reduces to the problem of evaluating the distribution of $D_{l, m}^{(n)} \mid \boldsymbol{X}_{n}$ by combining (11) and (12) with the distributions of $K_{m}^{(n)} \mid \boldsymbol{X}_{n}$ and $M_{l, m}^{(n)} \mid \boldsymbol{X}_{n}$ recalled in the web appendix. While the distribution of $D_{l, m}^{(n)} \mid \boldsymbol{X}_{n}$ is explicit, in many situations of practical interest the additional sample size $m$ is required to be very large and the computational burden for evaluating this posterior distribution becomes overwhelming. This happens, for instance, in various genomic applications where one has to deal with relevant portions of cDNA libraries which typically consist of millions of genes. In this section, we show how to exploit the large $m$ asymptotic behavior of $D_{l, m}^{(n)} \mid \boldsymbol{X}_{n}$ in order to associate asymptotic credible intervals to the estimator $\hat{\mathcal{D}}_{n, m}(l)$.

Let $\boldsymbol{X}_{n}$ be a sample from $P_{\sigma, \theta}$ featuring $K_{n}=k_{n}$ species $X_{1}^{*}, \ldots, X_{K_{n}}^{*}$ with frequencies $\left(N_{1, n}, \ldots, N_{K_{n}, n}\right)=$ $\left(n_{1, n}, \ldots, n_{k_{n}, n}\right)$. Let $Z_{\sigma, \theta, k_{n}}^{(n)} \stackrel{\mathrm{d}}{=} B_{k_{n}+\theta / \sigma, n / \sigma-k_{n}} Z_{\sigma,(\theta+n) / \sigma}$ where $B_{a, b}$ is a Beta random variable with parameter $(a, b)$ and $Z_{\sigma, q}$ has density function $f_{Z_{\sigma, q}}(z)=\Gamma(q \sigma+1) z^{q-1-1 / \sigma} f_{\sigma}\left(z^{-1 / \sigma}\right) / \sigma \Gamma(q+$
$D_{\left(l_{1}, \ldots, l_{\tau}\right), m}^{(n)}=\sum_{1 \leq i \leq \tau} D_{l_{i}, m}^{(n)}$. The distribution takes on the interpretation of the posterior $d$ $\left(m ; l_{1}, \ldots, l_{\tau}\right)$-discovery. In the next proposit the fluctuation limit (15) to the cumulative ity $D_{\left(l_{1}, \ldots, l_{\tau}\right), m}^{(n)} \mid \boldsymbol{X}_{n}$.

Proposition 1. Let $\boldsymbol{X}_{n}$ be a sample of siz turing $K_{n}=k_{n}$ species with corresponding $\left(M_{1, n}, \ldots, M_{n, n}\right)=\left(m_{1, n}, \ldots, m_{n, n}\right)$. Then, a has

$$
\frac{D_{\left(l_{1}, \ldots, l_{\tau}\right), m}^{(n)}}{m^{\sigma-1}} \mid \boldsymbol{X}_{n} \stackrel{w}{\longrightarrow}\left(\sum_{i=1}^{\tau} \frac{\sigma(1-\sigma)_{l_{i}}}{l_{i} !}\right)
$$

Fluctuation limits (15) and (16) provide $\mathrm{u}$ proximating the distribution of $D_{l, m}^{(n)} \mid \boldsymbol{X}_{n}$ an The same fluctuation limits hold for any sc such that, as $m \rightarrow+\infty, r(m) \simeq m^{\sigma-1}$. This a duce a scaling factor finer than $m^{\sigma-1}$. Inde ily verified that, as soon as $\theta$ and $n$ are no smaller than $m$,

$$
\hat{\mathcal{D}}_{n, m}^{\prime}(l)=m^{\sigma-1} \frac{\sigma(1-\sigma)_{l}}{l !} \mathbb{E}\left[Z_{\sigma, \theta, k_{l}}^{(n)}\right.
$$

with $\mathbb{E}\left[Z_{\sigma, \theta, k_{n}}^{(n)}\right]=\left(k_{n}+\theta / \sigma\right) \Gamma(\theta+n) / \Gamma(\theta+n$ from $\hat{\mathcal{D}}_{n, m}(l)$. Hence, the corresponding ible intervals could be far from the Of course, the same issue appears for $\hat{\mathcal{D}}_{n, m}\left(l_{1}, \ldots, l_{\tau}\right)$. For this reason we cons factors $r^{*}(m, l)$ and $r^{*}\left(m, l_{1}, \ldots, l_{\tau}\right)$ in $\mathrm{su}$ $\hat{\mathcal{D}}_{n, m}(l)=r^{*}(m, l)\left(\sigma(1-\sigma)_{l} / l !\right) \mathbb{E}\left[Z_{\sigma, \theta, k_{n}}^{(n)}\right]$ and

$$
\begin{array}{r}
r^{*}\left(m, l_{1}, \ldots, l_{\tau}\right) \sum_{1 \leq i \leq \tau}\left(\sigma(1-\sigma)_{l_{i}} / l_{i} !\right) \mathbb{E}\left[Z_{\sigma, \theta, k_{n}}^{(n)}\right. \\
\hat{\mathcal{D}}_{n, m}^{*}(l)=r^{*}(m, l) \frac{\sigma(1-\sigma)_{l}}{l !} \mathbb{E}\left[Z_{\sigma, \theta},\right.
\end{array}
$$

and

$\hat{\mathcal{D}}_{n, m}^{*}\left(l_{1}, \ldots, l_{\tau}\right)=r^{*}\left(m, l_{1}, \ldots, l_{\tau}\right)\left(\sum_{i=1}^{\tau} \frac{\sigma(1-}{l_{i}}\right.$

It can be easily verified that, as $m \rightarrow+\infty$ and $r^{*}\left(m, l_{1}, \ldots, l_{\tau}\right) \simeq m^{\sigma-1}$. Explicit expres ing factors $r^{*}(m, l)$ and $r^{*}\left(m, l_{1}, \ldots, l_{\tau}\right)$ are appendix. The reader is referred to Favaro 
$\left(r^{*}(m, l) \sigma(1-\sigma)_{l} s_{1} / l !, r^{*}(m, l) \sigma(1-\sigma)_{l} s_{2} / l !\right)$ is a $95 \%$ asymptotic credible interval for $\hat{\mathcal{D}}_{n, m}(l)$. Analogous observations hold true for the estimator $\hat{\mathcal{D}}_{n, m}\left(l_{1}, \ldots, l_{\tau}\right)$. In order to determine the quantiles $s_{1}$ and $s_{2}$, we resort to a simulation algorithm for sampling the limiting random variable $Z_{\sigma, \theta, k_{n}}^{(n)}$. Note that, according to the definition of $Z_{\sigma, \theta, k_{n}}^{(n)}$, this procedure involves sampling from the random variable $Z_{\sigma, q}$ with density function $f_{Z_{\sigma, q}}(z)=\Gamma(q \sigma+1) z^{q-1-1 / \sigma} f_{\sigma}\left(z^{-1 / \sigma}\right) / \sigma \Gamma(q+1)$.

A strategy for sampling $Z_{\sigma, q}$ was proposed by Favaro et al. (2009). Specifically, let $L_{\sigma, q}=Z_{\sigma, q}^{-1 / \sigma}$ and we introduce a Gamma random variable $U_{q}$ with parameter $(q, 1)$. Then, conditionally on $U_{q}=u$, the distribution of $L_{\sigma, q}$ has density function proportional to $f_{\sigma}(x) \exp \{-u x\}$. Therefore, the problem of sampling from $Z_{\sigma, q}$ boils down to the problem of sampling from an exponentially tilted stable distribution. Here we improve the sampling scheme proposed in Favaro et al. (2009) by resorting to the fast rejection algorithm recently proposed in Hofert (2011) for sampling from an exponentially tilted positive $\sigma$-stable random variable. Summarizing, in order to generate random variates from the distribution of $Z_{\sigma, \theta, k_{n}}^{(n)}$, we have the following steps: (i) sample $B_{k_{n}+\theta / \sigma, n / \sigma-k_{n}}$; (ii) sample $G_{(\theta+n) / \sigma, 1}$ and set $U_{(\theta+n) / \sigma}=G_{(\theta+n) / \sigma, 1}^{1 / \sigma}$; (iii) given $U_{(\theta+n) / \sigma}=u$, sample $L_{\sigma,(\theta+n) / \sigma}$ from density proportional to $f_{\sigma}(x) \exp \{-u x\}$, by means of the fast rejection sampling, and set $Z_{\sigma,(\theta+n) / \sigma}=$ $L_{\sigma,(\theta+n) / \sigma}^{-\sigma}$; (iv) set $Z_{\sigma, \theta, k_{n}}^{(n)}=B_{k_{n}+\theta / \sigma, n / \sigma-k_{n}} Z_{\sigma,(\theta+n) / \sigma}$.

\section{Illustrations}

In order to implement our results, the first issue to be faced is the specification of the parameter $(\sigma, \theta)$ in the $\operatorname{PD}(\sigma, \theta)$ prior. Hereafter, following the approach of Lijoi et al. (2007) and Favaro et al. (2012), we resort to an empirical Bayes procedure. Specifically let $\boldsymbol{X}_{n}$ be a sample from $P_{\sigma, \theta}$ featuring $K_{n}=$ $k_{n}$ species with frequencies $\left(N_{1, n}, \ldots, N_{K_{n}, n}\right)=\left(n_{1, n}, \ldots, n_{k_{n}, n}\right)$. The empirical Bayes procedure consists in choosing $\theta$ and $\sigma$ that maximize the distribution of $\boldsymbol{X}_{n}$. This, under a $\operatorname{PD}(\sigma, \theta)$ prior, corresponds to setting $(\sigma, \theta)=(\hat{\sigma}, \hat{\theta})$, where

$$
(\hat{\sigma}, \hat{\theta})=\underset{(\sigma, \theta)}{\arg \max }\left\{\frac{\prod_{i=0}^{k_{n}-1}(\theta+i \sigma)}{(\theta)_{n}} \prod_{i=1}^{k_{n}}(1-\sigma)_{n_{i, n}-1}\right\} .
$$

One could also specify a prior distribution on the parameter $(\sigma, \theta)$ and then seek a full Bayesian inference. However, in terms of estimating $D_{n, m}(l)$, there are no relevant differences between this fully Bayes approach and the empirical Bayes approach, given the posterior distribution of $(\sigma, \theta)$ is highly concentrated; this is typically the case of large datasets since
We compare the performance of the $I$ ric estimators for the $(0 ; l)$-discovery w responding Good-Turing estimators Turing estimators, for some choices o We draw 500 samples of size $n=1000 \mathrm{fr}$ with scale parameter $s=1.5$. Recall the able $Z$ is such that $\mathbb{P}[Z=z]=z^{-s} / C(s)$ for $s>1$. Next we order the samples ac of observed distinct species $k_{n}$ and we s Specifically, for $i=1,2, \ldots, 5$, the $i$-th be composed by 100 samples featuring served distinct species $k_{n}$ that stays be order $(i-1) / 5$ and $i / 5$ of the empirical therefore pick at random one sample fo it with the corresponding index $i$. Thi total number of 5 samples of 1000 obse species compositions.

We use these simulated datasets mators for the $(0 ; l)$-discovery with $D_{n, 0}(l)$, for $l=0,1,5,10,20,30$. Specif Bayesian nonparametric estimator $\hat{\mathcal{D}}_{n, \mathrm{c}}$ estimator $\check{\mathcal{D}}_{n, 0}(l)$, the smoothed Gc $\breve{\mathcal{D}}_{n, 0}\left(l ; \mathscr{S}_{\mathrm{PD}}\right)$, and the Poisson smoothed tor $\breve{\mathcal{D}}_{n, 0}\left(l ; \mathscr{S}_{\mathrm{Poi}}\right)$ with $\tau=1$ and $\lambda=n / k$ sider the so-called Simple Good-Turi by $\check{\mathcal{D}}_{n, 0}\left(l ; \mathscr{S}_{\mathrm{SGT}}\right)$, which is a popular sr estimator discussed in Chapter 7 of Sa ically, in the Simple Good-Turing est rule $\mathscr{S}_{\text {SGT }}$ consists in first computing, ues $z_{l, n}$ that take into account both $\mathrm{t}$ counts $m_{l, n}$ and the surrounding zero v sorting to a line of best fit for the pair in order to obtain the smoothed values

Table 1 summarizes the result of or As an overall measure for the perfor tors, we use the sum of squared error generic estimator $\hat{D}(l)$ of the $(0, l)$-disc $\sum_{0 \leq l \leq n}\left(\hat{D}(l)-d_{n, 0}(l)\right)^{2}$, with $d_{n, 0}(l)$ be $D_{n, 0}(\bar{l})$. By looking at the SSE in Tabl $\hat{\mathcal{D}}_{n, 0}(l)$ and $\check{\mathcal{D}}_{n, 0}\left(l ; \mathscr{S}_{\mathrm{SGT}}\right)$ are much $\mathrm{m}$ others. As expected, the Good-Turing good performance only for small values cies arise for large frequencies thus exp of the resulting SSE. For instance, since one species that has frequency $l=20$ frequency $l=21$, the Good-Turing est 0 while, clearly, there is positive prob species appeared 20 times in the sample yields a smaller SSE than $\check{\mathcal{D}}_{n, 0}\left(l ; \mathscr{S}_{\text {Poi }}\right)$. curacy of $\check{\mathcal{D}}_{n, 0}\left(l ; \mathscr{S}_{\mathrm{PD}}\right)$ and $\tilde{\mathcal{D}}_{n, 0}\left(l ; \mathscr{S}_{\text {Poi }}\right.$ and $\check{\mathcal{D}}_{n, 0}\left(l ; \mathscr{S}_{\mathrm{SGT}}\right)$, shows that the pa 


\begin{tabular}{|c|c|c|c|c|c|}
\hline \multirow{5}{*}{$l=0$} & $\hat{\mathcal{D}}_{n, 0}(l)$ & 0.0871 & 0.0939 & 0.1004 & 0.1016 \\
\hline & $\check{\mathcal{D}}_{n, 0}(l)$ & 0.0870 & 0.0950 & 0.1040 & 0.1040 \\
\hline & $\check{\mathcal{D}}_{n, 0}\left(l ; \mathscr{S}_{\mathrm{Poi}}\right)$ & 0.0006 & 0.0008 & 0.0008 & 0.0011 \\
\hline & $\check{\mathcal{D}}_{n, 0}\left(l ; \mathscr{S}_{\mathrm{PD}}\right)$ & 0.0859 & 0.0933 & 0.1002 & 0.1011 \\
\hline & $\check{\mathcal{D}}_{n, 0}\left(l ; \mathscr{S}_{\mathrm{SGT}}\right)$ & 0.0870 & 0.0950 & 0.1040 & 0.1040 \\
\hline \multirow{6}{*}{$l=1$} & $D_{n, 0}(l)$ & 0.0273 & 0.0272 & 0.0478 & 0.0365 \\
\hline & $\hat{\mathcal{D}}_{n, 0}(l)$ & 0.0320 & 0.0312 & 0.0301 & 0.0319 \\
\hline & $\check{\mathcal{D}}_{n, 0}(l)$ & 0.0320 & 0.0220 & 0.0160 & 0.0240 \\
\hline & $\check{\mathcal{D}}_{n, 0}\left(l ; \mathscr{S}_{\mathrm{Poi}}\right)$ & 0.0047 & 0.0054 & 0.0059 & 0.0073 \\
\hline & $\check{\mathcal{D}}_{n, 0}\left(l ; \mathscr{S}_{\mathrm{PD}}\right)$ & 0.0316 & 0.0307 & 0.0290 & 0.0311 \\
\hline & $\check{\mathcal{D}}_{n, 0}\left(l ; \mathscr{S}_{\mathrm{SGT}}\right)$ & 0.0319 & 0.0221 & 0.0161 & 0.0240 \\
\hline \multirow{6}{*}{$l=5$} & $D_{n, 0}(l)$ & 0.0060 & 0.0238 & 0.0132 & 0.0154 \\
\hline & $\hat{\mathcal{D}}_{n, 0}(l)$ & 0.0044 & 0.0173 & 0.0086 & 0.0215 \\
\hline & $\check{\mathcal{D}}_{n, 0}(l)$ & 0.0240 & 0.0180 & 0.0120 & 0.0180 \\
\hline & $\check{\mathcal{D}}_{n, 0}\left(l ; \mathscr{S}_{\mathrm{Poi}}\right)$ & 0.1148 & 0.1206 & 0.1243 & 0.1332 \\
\hline & $\check{\mathcal{D}}_{n, 0}\left(l ; \mathscr{S}_{\mathrm{PD}}\right)$ & 0.0126 & 0.0114 & 0.0101 & 0.0111 \\
\hline & $\check{\mathcal{D}}_{n, 0}\left(l ; \mathscr{S}_{\mathrm{SGT}}\right)$ & 0.0044 & 0.0176 & 0.0089 & 0.0219 \\
\hline \multirow{6}{*}{$l=10$} & $D_{n, 0}(l)$ & 0.0105 & 0 & 0.0105 & 0.0092 \\
\hline & $\hat{\mathcal{D}}_{n, 0}(l)$ & 0.0094 & 0 & 0.0093 & 0.0093 \\
\hline & $\check{\mathcal{D}}_{n, 0}(l)$ & 0 & 0 & 0.0220 & 0.0110 \\
\hline & $\check{\mathcal{D}}_{n, 0}\left(l ; \mathscr{S}_{\mathrm{Poi}}\right)$ & 0.0816 & 0.0769 & 0.0738 & 0.0664 \\
\hline & $\check{\mathcal{D}}_{n, 0}\left(l ; \mathscr{S}_{\mathrm{PD}}\right)$ & 0.0082 & 0.0072 & 0.0062 & 0.0070 \\
\hline & $\check{\mathcal{D}}_{n, 0}\left(l ; \mathscr{S}_{\mathrm{SGT}}\right)$ & 0.0093 & 0 & 0.0094 & 0.0093 \\
\hline \multirow{6}{*}{$l=20$} & $D_{n, 0}(l)$ & 0 & 0.0142 & 0.0169 & 0 \\
\hline & $\hat{\mathcal{D}}_{n, 0}(l)$ & 0 & 0.0193 & 0.0193 & 0 \\
\hline & $\check{\mathcal{D}}_{n, 0}(l)$ & 0 & 0 & 0 & 0 \\
\hline & $\check{\mathcal{D}}_{n, 0}\left(l ; \mathscr{S}_{\mathrm{Poi}}\right)$ & 0.0001 & 0.0000 & 0.0000 & 0.0000 \\
\hline & $\check{\mathcal{D}}_{n, 0}\left(l ; \mathscr{S}_{\mathrm{PD}}\right)$ & 0.0053 & 0.0046 & 0.0038 & 0.0043 \\
\hline & $\check{\mathcal{D}}_{n, 0}\left(l ; \mathscr{S}_{\mathrm{SGT}}\right)$ & 0 & 0.0194 & 0.0195 & 0 \\
\hline \multirow{6}{*}{$l=30$} & $D_{n, 0}(l)$ & 0.0260 & 0 & 0 & 0 \\
\hline & $\hat{\mathcal{D}}_{n, 0}(l)$ & 0.0293 & 0 & 0 & 0 \\
\hline & $\check{\mathcal{D}}_{n, 0}(l)$ & 0 & 0 & 0 & 0 \\
\hline & $\check{\mathcal{D}}_{n, 0}\left(l ; \mathscr{S}_{\mathrm{Poi}}\right)$ & 0.0000 & 0.0000 & 0.0000 & 0.0000 \\
\hline & $\check{\mathcal{D}}_{n, 0}\left(l ; \mathscr{S}_{\mathrm{PD}}\right)$ & 0.0041 & 0.0035 & 0.0029 & 0.0033 \\
\hline & $\check{\mathcal{D}}_{n, 0}\left(l ; \mathscr{S}_{\mathrm{SGT}}\right)$ & 0.0292 & 0 & 0 & 0 \\
\hline \multicolumn{2}{|c|}{$\operatorname{MSE}\left(\hat{\mathcal{D}}_{n, 0}\right)$} & 0.0006 & 0.0016 & 0.0007 & 0.0007 \\
\hline \multicolumn{2}{|c|}{$\operatorname{MSE}\left(\check{\mathcal{D}}_{n, 0}\right)$} & 0.3475 & 0.3773 & 0.3460 & 0.3575 \\
\hline \multicolumn{2}{|c|}{$\operatorname{MSE}\left(\check{\mathcal{D}}_{n, 0}\left(\mathscr{S}_{\text {Poi }}\right)\right)$} & 0.2657 & 0.2723 & 0.2765 & 0.2769 \\
\hline \multicolumn{2}{|c|}{$\operatorname{MSE}\left(\mathcal{\mathcal { D }}_{n, 0}\left(\mathscr{S}_{\mathrm{PD}}\right)\right)$} & 0.1748 & 0.1748 & 0.1753 & 0.1746 \\
\hline \multicolumn{2}{|c|}{$\operatorname{MSE}\left(\check{\mathcal{D}}_{n, 0}\left(\mathscr{S}_{\mathrm{SGT}}\right)\right)$} & 0.0007 & 0.0018 & 0.0014 & 0.0008 \\
\hline
\end{tabular}

underlying the smoothing rules $\mathscr{S}_{\text {Poi }}$ and $\mathscr{S}_{\text {PD }}$ are not suitable for data generated according to a Zeta distribution.

4.2. Credible Intervals for $\hat{\mathcal{D}}_{n, m}\left(l_{1}, \ldots, l_{\tau}\right)$

We illustrate the implementation of the asymptotic credible intervals for the Bayesian nonparametric estimator
$\hat{\mathcal{D}}_{n, m}\left(l_{1}, \ldots, l_{\tau}\right)$ through the analysis of EST by sequencing a benchmark cDNA library. an efficient way to characterize expressed $g$ ganism. The rate of gene discovery depends redundancy of the cDNA library from whic are obtained. Correctly estimating the relati 


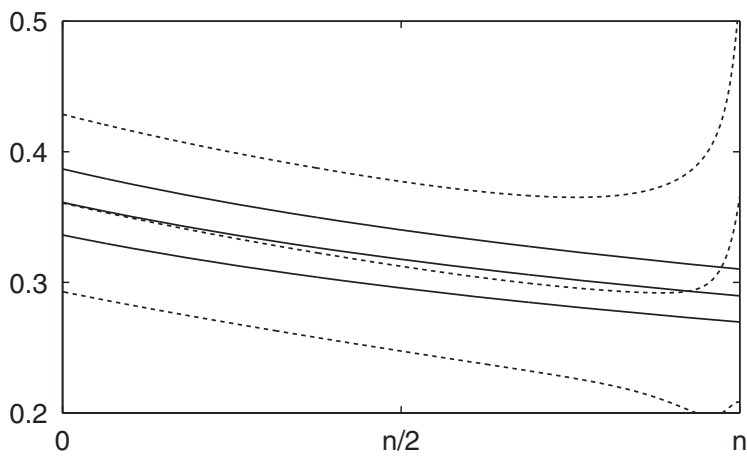

(a) Naegleria Aerobic

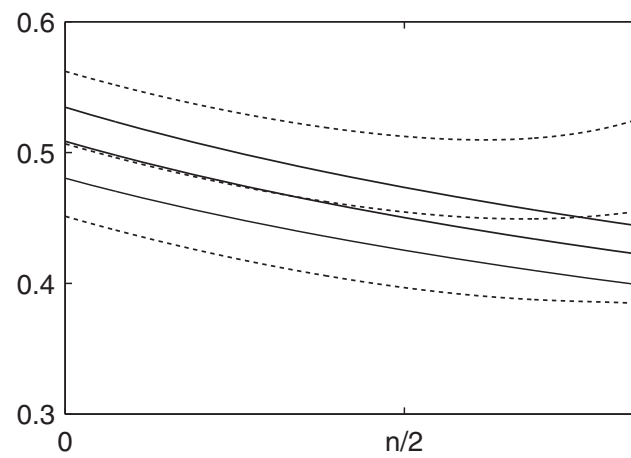

(b) Naegleria Anaerobic

Figure 2. Comparison of Good-Toulmin estimator $\check{\mathcal{D}}_{n, m}(0)$ (inner dashed curves) and Bayesian nonpara $\hat{\mathcal{D}}_{n, m}(0)$ (inner solid curves) for $m$ ranging in $[0, n]$. The Good-Toulmin estimates are endowed with $95 \%$ col (outer dashed curves). Bayesian nonparametric estimators are endowed with asymptotic $95 \%$ credible inte curves).

Table 3

Naegleria aerobic and Naegleria anaerobic libraries. $\hat{\mathcal{D}}_{n, m}(l)$, for $l=0,1,2,3,4$, and $\hat{\mathcal{D}}_{n, m}(0, \ldots, \tau)$, for $\tau$ corresponding asymptotic $95 \%$ credible intervals (c.i.)

\begin{tabular}{|c|c|c|c|c|c|c|}
\hline & \multirow[b]{2}{*}{ Library } & \multicolumn{2}{|c|}{$m=n$} & \multicolumn{2}{|c|}{$m=2 n$} & \multirow[b]{2}{*}{ estimate } \\
\hline & & estimate & $95 \%$ c.i. & estimate & $95 \%$ c.i. & \\
\hline$(m ; 0)$-discovery & $\begin{array}{l}\text { aerobic } \\
\text { anaerobic }\end{array}$ & $\begin{array}{l}0.289 \\
0.409\end{array}$ & $\begin{array}{l}(0.267,0.312) \\
(0.387,0.431)\end{array}$ & $\begin{array}{l}0.253 \\
0.358\end{array}$ & $\begin{array}{l}(0.234,0.273) \\
(0.339,0.378)\end{array}$ & $\begin{array}{l}0.231 \\
0.326\end{array}$ \\
\hline$(m ; 1)$-discovery & $\begin{array}{l}\text { aerobic } \\
\text { anaerobic }\end{array}$ & $\begin{array}{l}0.093 \\
0.130\end{array}$ & $\begin{array}{l}(0.084,0.101) \\
(0.123,0.137)\end{array}$ & $\begin{array}{l}0.083 \\
0.117\end{array}$ & $\begin{array}{l}(0.076,0.089) \\
(0.111,0.124)\end{array}$ & $\begin{array}{l}0.075 \\
0.108\end{array}$ \\
\hline$(m ; 2)$-discovery & $\begin{array}{l}\text { aerobic } \\
\text { anaerobic }\end{array}$ & $\begin{array}{l}0.061 \\
0.080\end{array}$ & $\begin{array}{l}(0.057,0.066) \\
(0.076,0.085)\end{array}$ & $\begin{array}{l}0.054 \\
0.075\end{array}$ & $\begin{array}{l}(0.050,0.059) \\
(0.071,0.079)\end{array}$ & $\begin{array}{l}0.050 \\
0.070\end{array}$ \\
\hline$(m ; 3)$-discovery & $\begin{array}{l}\text { aerobic } \\
\text { anaerobic }\end{array}$ & $\begin{array}{l}0.046 \\
0.059\end{array}$ & $\begin{array}{l}(0.042,0.049) \\
(0.056,0.062)\end{array}$ & $\begin{array}{l}0.041 \\
0.055\end{array}$ & $\begin{array}{l}(0.038,0.045) \\
(0.052,0.058)\end{array}$ & $\begin{array}{l}0.038 \\
0.052\end{array}$ \\
\hline$(m ; 4)$-discovery & $\begin{array}{l}\text { aerobic } \\
\text { anaerobic }\end{array}$ & $\begin{array}{l}0.036 \\
0.045\end{array}$ & $\begin{array}{l}(0.033,0.039) \\
(0.042,0.047)\end{array}$ & $\begin{array}{l}0.034 \\
0.044\end{array}$ & $\begin{array}{l}(0.031,0.036) \\
(0.042,0.046)\end{array}$ & $\begin{array}{l}0.031 \\
0.042\end{array}$ \\
\hline$(m ; 0,1,2,3)$-discovery & $\begin{array}{l}\text { aerobic } \\
\text { anaerobic }\end{array}$ & $\begin{array}{l}0.490 \\
0.679\end{array}$ & $\begin{array}{r}(0.452,0.528) \\
(0.642,0.716)\end{array}$ & $\begin{array}{l}0.432 \\
0.606\end{array}$ & $\begin{array}{l}(0.399,0.465) \\
(0.573,0.640)\end{array}$ & $\begin{array}{l}0.394 \\
0.556\end{array}$ \\
\hline $\begin{array}{l}(m ; 0,1,2,3,4) \text {-discovery } \\
(m ; 0,1,2,3,4,5) \text {-discovery }\end{array}$ & $\begin{array}{l}\text { aerobic } \\
\text { anaerobic } \\
\text { aerobic } \\
\text { anaerobic }\end{array}$ & $\begin{array}{l}0.526 \\
0.724 \\
0.556 \\
0.760\end{array}$ & $\begin{array}{l}(0.485,0.563) \\
(0.685,0.763) \\
(0.514,0.599) \\
(0.718,0.801)\end{array}$ & $\begin{array}{l}0.465 \\
0.650 \\
0.494 \\
0.686\end{array}$ & $\begin{array}{l}(0.430,0.501) \\
(0.615,0.686) \\
(0.456,0.532) \\
(0.649,0.723)\end{array}$ & $\begin{array}{l}0.425 \\
0.599 \\
0.452 \\
0.634\end{array}$ \\
\hline
\end{tabular}


tainty are provided for these estimates. In Table 3 we summarize estimates of the $(m ; l)$-discovery for $l=0, \ldots, 4$ and of the $\left(m ; l_{1}, \ldots, l_{\tau}\right)$-discovery for $\tau=3,4,5$. These estimates are endowed with asymptotic $95 \%$ credible intervals obtained by combining asymptotic results displayed in (15) and (16) with the choice of the scaling factors $r^{*}(m, l)$ and $r^{*}\left(m, l_{1}, \ldots, l_{\tau}\right)$, respectively. Table 3 thus complete the illustrations presented in Favaro et al. (2009) and Favaro et al. (2012).

\section{Supplementary Materials}

Web Appendices, Tables, and Figures referenced in Sections 2, 3 and 4 are available with this paper at the Biometrics website on Wiley Online Library. The Matlab code for computing the asymptotic credible intervals for $\hat{\mathcal{D}}_{n, m}\left(l_{1}, \ldots, l_{\tau}\right)$ is also available at the Biometrics website on Wiley Online Library.

\section{ACKNOWLEDGEMENTS}

The authors are grateful to an Associate Editor and an anonymous referee for their constructive comments and suggestions. Stefano Favaro is supported by the European Research Council through StG N-BNP 306406. Yee Whye Teh is supported by the European Research Council through the European Unions Seventh Framework Programme (FP7/20072013) ERC grant agreement 617411.

\section{REFERENCES}

Bunge, J. and Fitzpatrick, M. (1993). Estimating the number of species: A review. Journal of the American Statistical Association $\mathbf{8 8}, 364-373$.

Bunge, J., Willis, A., and Walsh, F. (2014). Estimating the number of species in microbial diversity studies. Annual Review of Statistics and Its Application 1, 427-445.

De Blasi, P., Favaro, S., Lijoi, A., Mena, R. H., Prünster, I., and Ruggiero, M. (2015). Are Gibbs-type priors the most natural generalization of the Dirichlet process? IEEE Trans-
Favaro, S., Lijoi, A., and Prünster, I. (20 the discovery probability. Biometric

Favaro, S., Lijoi, A., and Prünster, I. (201: for Gibbs-type exchangeable rando Applied Probability 23, 1721-1754.

Gnedin, S., Hansen, B., and Pitman, J. (2 pancy problem with infinitely many totics and power law. Probability $\mathrm{S}$

Good, I. J. (1953). The population freque estimation of population parameter 264.

Good, I. J. and Toulmin, G. H. (1956). The and the increase in population cove increased. Biometrika 43, 45-63.

Hofert, M. (2011). Efficiently sampling nes las. Computational Statistics \& Dat

Lijoi, A., Mena, R. H., and Prünster, I. parametric estimation of the probal species. Biometrika 94, 769-786.

Mao, C. X. (2004). Prediction of the condi covering a new class. Journal of $t$ Association 99, 1108-1118.

Pitman, J. (1995). Exchangeable and par dom partitions. Probability Theory $145-158$.

Pitman, J. (2003). Poisson-Kingman partit tics: A Festschrift for Terry Speed Lecture Notes Monograph Series $\mathbf{4 0}$ $\mathrm{OH}$.

Pitman, J. (2006). Combinatorial Stochast de Probabilités de Saint-Flour XX Mathematics N. 1875. New York: $\mathrm{S}_{\mathrm{I}}$

Sampson, G. (2001). Empirical Linguistic New York

Susko, E. and Roger, A. J. (2004). Estima rates of gene discovery and express frequencies in EST surveys. Bioinfo

Received October 2014. Revise Accepted June 201 